\title{
Dark Energy and the Return of the Phoenix Universe
}

\author{
Jean-Luc Lehners ${ }^{1}$ and Paul J. Steinhardt ${ }^{1,2}$ \\ ${ }^{1}$ Princeton Center for Theoretical Science, Princeton University, Princeton, NJ 08544 USA \\ ${ }^{2}$ Joseph Henry Laboratories, Princeton University, Princeton, NJ 08544, USA
}

\begin{abstract}
In cyclic universe models based on a single scalar field (e.g., the radion determining the distance between branes in M-theory), virtually the entire universe makes it through the ekpyrotic smoothing and flattening phase, bounces, and enters a new epoch of expansion and cooling. This stable evolution cannot occur, however, if scale-invariant curvature perturbations are produced by the entropic mechanism because it requires two scalar fields (e.g., the radion and the Calabi-Yau dilaton) evolving along an unstable classical trajectory. In fact, we show here that an overwhelming fraction of the universe fails to make it through the ekpyrotic phase; nevertheless, a sufficient volume survives and cycling continues forever provided the dark energy phase of the cycle lasts long enough, of order a trillion years. Two consequences are a new role for dark energy and a global structure of the universe radically different from that of eternal inflation.
\end{abstract}

Lemaitre [1] invoked the mythical "phoenix" to refer to an oscillatory cosmology in which the universe undergoes regular periods of expansion and contraction with a big crunch/big bang transition in between. His model was a closed universe in which the reversal from expansion to contraction is caused by an overdensity of matter and radiation. Although this model is ruled out by WMAP measurements [2] indicating a flat universe, there remains the more recent proposal of a cyclic model of the universe [3, 4] (see [5] for a recent review) that also undergoes regular cycles of evolution beginning with a big bang and ending in a big crunch. Unlike Lemaitre's model, the cyclic universe is kept smooth and spatially flat by a period of ekpyrotic contraction [6, 7] (slow contraction with equation of state $w \gg 1$ ) that precedes each big crunch. The expansion, contraction and smoothing are governed by a scalar field $\phi_{1}$ that evolves along a potential $V\left(\phi_{1}\right)$. In a version inspired by M theory [8] , the cycles correspond to the regular collision and rebound of two orbifold planes, and $\phi_{1}$ is the modulus field whose value determines the distance between orbifold planes. As in Lemaitre's oscillatory model, the notion is that virtually the entire universe makes it through the big crunch/big bang transition and continues into the next cycle.

In this paper, we show how the generation of scaleinvariant density perturbations may lead to a variation of the cyclic model that may be more fittingly called a "phoenix universe," in the sense that most of the universe is turned to "ash" at the end of each cycle and the universe is reborn from a comparatively tiny surviving seed. The phoenix effect is an unintended byproduct of generating curvature perturbations via the entropic mechanism [9]. The entropic mechanism was introduced because an ekpyrotic contraction phase with only a single scalar field produces scale-invariant time-delay perturbations, but not scale-invariant curvature perturbations 10]. Although there are various proposals for circumventing this problem [9, 11], the entropic mechanism is currently the best understood and can be fully analyzed in four dimensional field theory without invoking extra dimensions [9, 10, 12, 13]. The mechanism relies on having two scalar fields (e.g., the radion and the Calabi-Yau dilaton [9]) each obtain scale-invariant fluctuations by evolving down a steep potential $V\left(\phi_{1}, \phi_{2}\right)$ during the ekpyrotic phase, with one linear combination forming a spectrum of time-delay fluctuations and the second a spectrum of entropic perturbations. The latter are converted to curvature fluctuations if the two-field trajectory bends or bounces as the universe approaches the big crunch, which occurs generically.

An essential feature of the entropic mechanism is that the classical ekpyrotic trajectory along $V\left(\phi_{1}, \phi_{2}\right)$ is unstable to transverse fluctuations [9, 10, 12, 13]. A consequence is that, without adding some mechanism to force the universe to begin very close to the classical track [13], an overwhelming fraction of the universe fails to make it all the down the classical trajectory simply due to quantum fluctuations. This fraction is transformed into highly inhomogeneous remnants and black holes that do not cycle or grow in the post-big bang phase. However, as shown below, something curious happens if the dark energy expansion phase preceding the ekpyrotic contraction phase lasts at least 600 billion years. Then, a sufficiently large patch of space makes it all the way down the classical trajectory and through the big bang such that, fourteen billion years later, it comprises the overwhelming majority of space. This surviving volume, which grows in absolute size from cycle to cycle, consists of a smooth, flat, expanding space with nearly scale-invariant curvature perturbations, in accordance with what is observed today. As with the mythical phoenix, a new habitable universe grows from the ashes of the old.

The phoenix picture is in some sense minimalist. No features have to be added to the cyclic model that were not already there, e.g., in the original version inspired by $M$ theory and the Hořava-Witten model [8]. One only has had to appreciate the effects of the elements that were in place. The heterotic M-theory embedding of the cyclic model [14] already admits a four-dimensional effective description in terms of gravity coupled to two canonically normalized scalar fields. The scalars parameterize geometrical quantities in the higher-dimensional theory, namely the distance between the boundary branes 
and the volume of the internal Calabi-Yau manifold. Also, dark energy was always incorporated into the cyclic model to stabilize the oscillatory behavior. Here dark energy is given the added role of ensuring the survival of the phoenix universe. The result is a universe with a global structure dramatically different from eternal inflation and a history somewhat different from earlier oscillatory and cyclic universes.

For concreteness, we will develop the phoenix picture based on the Hořava-Witten model [8] so that there is simultaneously a $4 \mathrm{~d}$ and higher dimensional interpretation. In this picture, the relevant degrees of freedom aside from the two scalars is the radiation (with energy density $\rho_{ \pm}$) produced on the positive and negative tension branes respectively, at the big crunch/big bang transition; it appears in the effective theory with a coupling $\beta_{ \pm}$that depends on the scalar fields [4]. Thus the equations of motion are

$$
\begin{aligned}
& 3 H^{2}=\frac{1}{2}\left(\dot{\phi}_{1}^{2}+\dot{\phi}_{2}^{2}\right)+V\left(\phi_{i}\right)+\beta_{+}^{4}\left(\phi_{i}\right) \rho_{+}+\beta_{-}^{4}\left(\phi_{i}\right) \rho_{-} \\
& \ddot{\phi}_{i}+3 H \dot{\phi}_{i}+V_{, \phi_{i}}=0 \\
& 3 \frac{\ddot{a}}{a}=-\left(\dot{\phi}_{1}^{2}+\dot{\phi}_{2}^{2}\right)+V\left(\phi_{i}\right)-\beta_{+}^{4}\left(\phi_{i}\right) \rho_{+}-\beta_{-}^{4}\left(\phi_{i}\right) \rho_{-},
\end{aligned}
$$

where we have assumed a Friedmann-Robertson-Walker background with line element $\underline{s}^{2}=-t^{2}+a^{2}(t) \mathbf{x}^{2} ; a(t)$ denoting the scale factor and $H \equiv \dot{a} / a$, with ${ }^{\circ} \equiv \frac{\partial}{\partial t}$. These equations form a closed system subject to the equation of continuity

$$
\frac{\partial}{\partial t}\left(\beta_{ \pm}^{4} \rho_{ \pm}\right)+4 H \beta_{ \pm}^{4} \rho_{ \pm}=0
$$

which implies that

$$
\beta_{+}^{4} \rho_{+}+\beta_{-}^{4} \rho_{-}=\frac{\rho_{0}}{a^{4}} .
$$

Hence we can treat all radiation as one, without the need to know the precise functional form of $\beta_{ \pm}$.

The effective theory contains a potential that is the sum of two parts,

$$
V=V_{e k}\left(\phi_{1}, \phi_{2}\right)+V_{r e p}\left(\phi_{2}\right) .
$$

The repulsive potential $V_{\text {rep }}$ reflects the fact that there is a boundary to scalar field space at $\phi_{2}=0$. This boundary corresponds to the negative-tension brane being repelled by a naked singularity in the higher-dimensional bulk spacetime, and the precise form of the potential depends on the nature of the matter living on the negative-tension brane (e.g. scalar matter or a perfect fluid) and on its coupling to the Calabi-Yau volume modulus; see [14, 15] for details. A simple form for the repulsive potential is $V_{\text {rep }} \propto \phi_{2}^{-2}$, but we have checked that our results are also valid for other functional forms of $V_{\text {rep }}$ arising in this way. The ekpyrotic potential is the two-field version of the cyclic potential described in [4]:

$$
V_{e k}=-V_{1} e^{-c_{1} \phi_{1}}-V_{2} e^{-c_{2} \phi_{2}}+V_{0}
$$

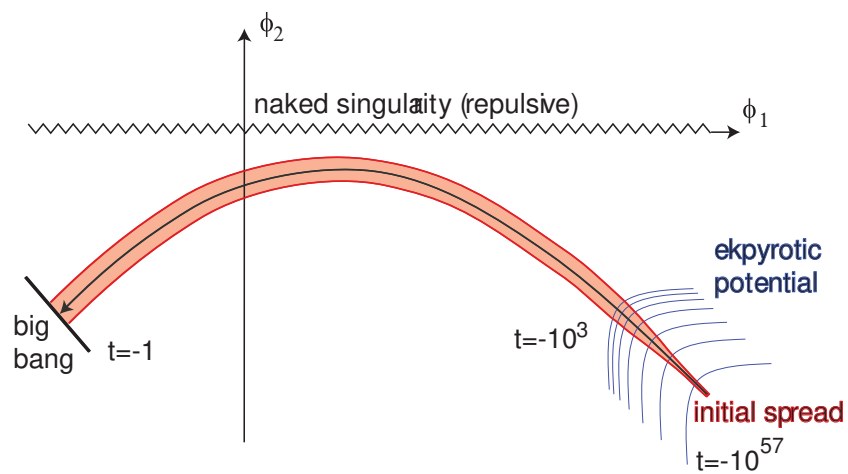

FIG. 1: This figure shows the evolution of adjacent classical trajectories from the ekpyrotic phase through a kinetic phase to the big crunch. The trajectories get reflected by a boundary in scalar field space at $\phi_{2}=0$. All times are in units of the Planck time.

The essential features are that the potential is steep and negative, but with a positive plateau at large field values. Here $V_{0}, V_{i}, c_{i} \gg 1$ are constants, although in general a small dependence of the $c_{i}$ on the fields $\phi_{i}$ is both natural to expect and necessary to obtain a spectrum with a small red tilt [9]. For the purposes of this paper we can restrict ourselves to considering constant $c_{i}$. During the ekpyrotic phase, the background trajectory is given by the scaling solution

$$
a=(-t)^{p}, \quad \phi_{i}=\frac{2}{c_{i}} \ln \left(-\sqrt{c_{i}^{2} V_{i} / 2} t\right),
$$

where $t=0$ corresponds to the brane collision and $p \equiv 2 / c_{1}^{2}+2 / c_{2}^{2} \ll 1$ is the equivalent of the slow-roll parameter in inflation. The trajectory evolves along the ridge in the potential. This is most easily seen [13, 16] by changing variables to $\sigma$ (pointing along the direction of the background trajectory) and $s$ (pointing transverse to the trajectory):

$$
\sigma \equiv \frac{c_{2} \phi_{1}+c_{1} \phi_{2}}{\sqrt{c_{1}^{2}+c_{2}^{2}}}, \quad s \equiv \frac{c_{2} \phi_{2}-c_{1} \phi_{1}}{\sqrt{c_{1}^{2}+c_{2}^{2}}} .
$$

Fluctuations in $\sigma$ correspond to adiabatic perturbations, while fluctuations in $s$ are entropy fluctuations. During the ekpyrotic phase, the classical background trajectory must follow the ridge at $s=0$, and around this trajectory the potential can be expanded as

$$
V_{e k}=V_{0}-V_{0} e^{-\sqrt{2 / p} \sigma}\left(1+\frac{1}{p} s^{2}+\cdots\right),
$$

where (by a shift in $\phi_{1}$ ) we have chosen $V_{1}$ such that the prefactor of the second term is $V_{0}$ also. This expression for the potential clearly shows that the $s$ direction is unstable during ekpyrosis. Hence, unlike for the single-field cyclic model, the background solution is not an attractor, and it is not clear at first how cycling can remain viable. In order to appreciate the severity of the instability, it is 
useful to solve the equation of motion for $s$ in the vicinity of the ridge [13]. To leading order in $p$, it is given by

$$
\ddot{s}-\frac{2}{t^{2}} s=0,
$$

and subject to the boundary conditions $s\left(t_{e k-b e g}\right) \equiv$ $3 s_{0} /\left(2 t_{e k-b e g}\right), \dot{s}\left(t_{e k-b e g}\right)=0$, for some constant $s_{0}$, it is solved by

$$
s=s_{0}\left(\frac{1}{t}+\frac{t^{2}}{2 t_{e k-b e g}^{3}}\right)
$$

in other words $s$ grows like the Hubble parameter $H$. Now, the ekpyrotic phase lasts from $t_{e k-b e g}$ to $t_{e k-e n d} \equiv$ $e^{-N_{e k}} t_{e k-b e g}$ and, hence, the spread in $s$ grows by a factor $e^{N_{e k}} . N_{e k}$ denotes the number of e-folds of ekpyrosis, and in order for the cyclic potential to interpolate between the dark energy and the grand unified scales (reaching the grand unified scale is necessary for the perturbations to have the right amplitude [9]), we need $N_{e k} \approx 120$. Hence the instability causes $s$ to grow by a huge factor of about $e^{120}$. Of course this means that at the start of the ekpyrotic phase, the field $s$ must be localized close to the ridge with great precision. As is already clear from the form of the potential (10) and as previously derived in [13], being in the ekpyrotic phase until $t_{e k-e n d}$ requires $\left|s\left(t_{\text {ek-end }}\right)\right| \lesssim p$, which translates into

$$
\left|s\left(t_{e k-b e g}\right)\right| \lesssim p e^{-N_{e k}}
$$

in Planck units.

After ekpyrosis, the energy density is dominated by the scalar field kinetic energies. This kinetic phase lasts for about $10^{3}$ Planck times, during which time the trajectory bends due to the repulsive potential $V_{\text {rep }}$, see Fig. [1. We stop the evolution about one Planck time before the crunch, and resume the description at about one Planck time after the big bang, see Fig. 2. What we assume about the crunch/bang transition is that the scalar field velocities are reversed in direction, and that they are increased in magnitude by a small parameter $\chi$, defined as [4]

$$
\dot{\phi}_{i, b a n g} \equiv-\sqrt{1+\chi} \dot{\phi}_{i, \text { crunch }}
$$

At the brane collision, radiation and matter are produced. In fact, if there is slightly more radiation produced on the negative-tension brane than on the positivetension one, then the consequence is precisely a slight increase in the scalar field kinetic energies, as assumed above [4]. (Some forms of scalar couplings to the matter fields, $\beta_{ \pm}$, also lead to increased scalar field kinetic energies [4].) This extra kinetic energy helps to overcome the Hubble damping due to the radiation created at the bang. The constraint that we impose on the radiation is that it should not come to dominate until the $\sigma$ field has rolled back up the ekpyrotic potential. As shown in [4, 17] this translates into a constraint on the reheat

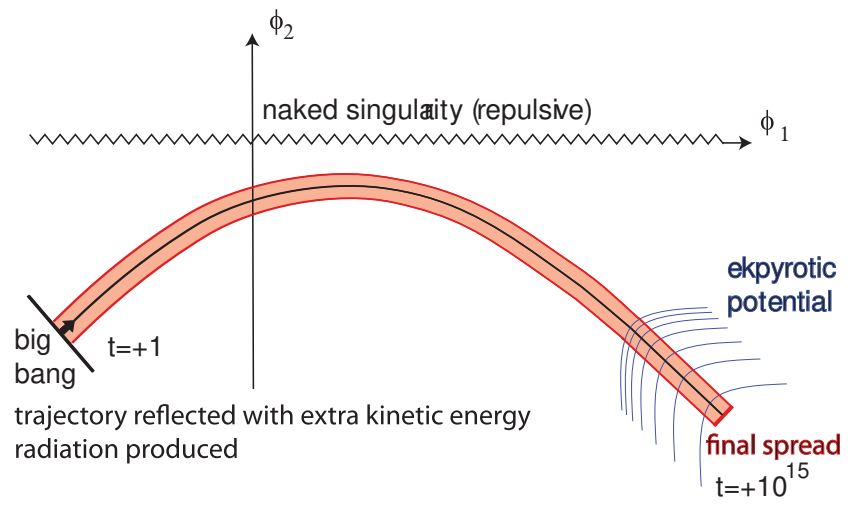

FIG. 2: After the big bang, adjacent trajectories proceed through a second kinetic phase and roll back up the ekpyrotic potential, where the radiation dominated phase starts at $t \approx 10^{15}$ Planck times. During the ensuing matter and dark energy phases, the scalar fields remain almost immobile.

temperature $T_{r}$

$$
T_{r} \leqslant\left(-V\left(t_{\text {ek-end }}\right)\right)^{1 / 4}\left|\frac{V_{0}}{V\left(t_{\text {ek-end }}\right)}\right| \sqrt{3 p / 16},
$$

which is easy to satisfy. Finally, we note that the radiation does not contribute to the repulsive potential $V_{\text {rep }}$ [15]. However, since there can be extra matter produced at the bang, which could increase the strength of $V_{\text {rep }}$, we have allowed for a fractional increase in $V_{\text {rep }}$ of (at most) $\chi$.

The extra kinetic energy of the scalars means that the $\sigma$ field quickly rolls back up the unstable ekpyrotic potential to the (almost-)plateau, and the trajectory deviates little from a straight line (note that the evolution is still kinetic dominated at this stage). Numerical simulations show that during the whole evolution after the ekpyrotic phase, the range of values of $s$ (which grows by a factor $e^{120}$ during the ekpyrotic phase) increases only by a factor of about 5. Also, the final range encompasses the initial one as long as $\sqrt{\chi} \lesssim 10^{-2}$, which is a reasonable assumption. Numerical simulations also show that the radiation and increase in $V_{\text {rep }}$ discussed above have a very small effect. Hence it is clear now that there is a fixed point to the entire evolution over the course of one cycle, and this fixed point is located exponentially close to the ridge. One might be worried that at the start of the dark energy phase, when the field finally turns around on the plateau of the potential, the trajectory might deviate substantially. This is avoided as long as the gradient of the potential in the $\sigma$ direction is much larger than in the $s$ direction, i.e. we must ensure that $\left|V_{, \sigma} / V_{, s}\right| \approx-\sqrt{p / 2} /|s| \gg 1$ or, in other words, we need to have $\left|s\left(t_{e k-b e g}\right)\right| \ll \sqrt{p}$. On account of (13), this is clearly satisfied.

So now consider a small range of $s$ around the fixed point: this range grows by about $e^{N_{e k}}$ over the course of one cycle, but, crucially, during this time the uni- 


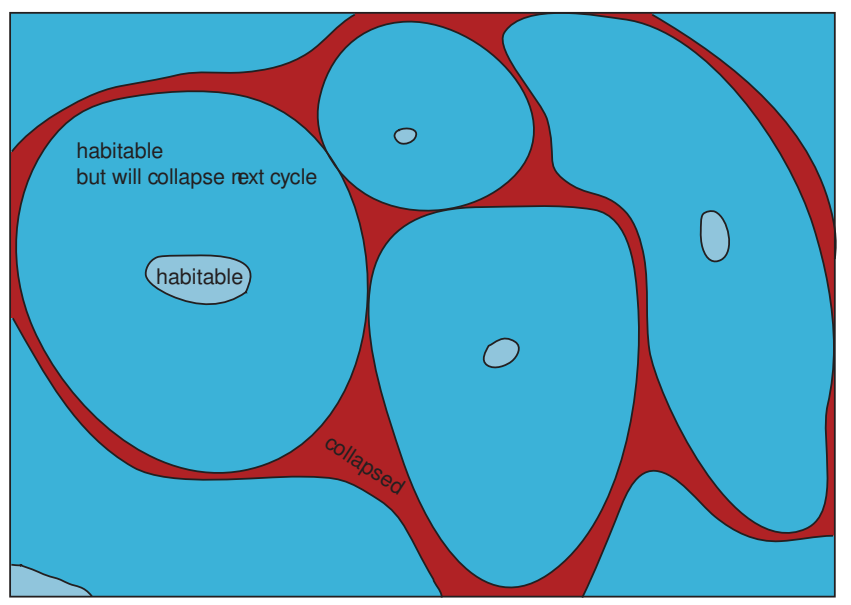

FIG. 3: The global structure of the two- field cyclic universe: large smooth and flat regions are interspersed with small regions that have collapsed and have stopped cycling. The tiny regions of lighter shade will turn into the entire habitable regions during the next cycle.

verse expands by a factor $e^{2 \gamma / 3+N_{\text {rad }}+N_{d e}}$, where $\gamma \equiv$ $\ln \left(\left|V\left(t_{\text {ek-end }}\right)\right|^{1 / 4} / T_{r}\right)$ parameterizes the total growth during the kinetic phases and $N_{r a d} \equiv \ln \left(T_{r} / T_{0}\right)$ is the number of e-folds of expansion between the start of radiation domination and today. Hence if

$$
N_{d e}>N_{e k}-\frac{2}{3} \gamma-N_{\text {rad }} \approx 60,
$$

then the gradient of values of $s$ is sufficiently diluted that a small initial flat patch will grow into a larger flat patch after each cycle. In other words, an initial patch of space, with a spread in field values $\Delta s$ around the fixed point value, will be amplified over the course of one cycle to a region containing a larger patch of space with the same spread $\Delta s$. Hence, starting from an arbitrarily small initial flat patch with the right values of $s$ one can cycle to a large, flat and habitable universe.

In principle, cycling can be eternal to the past and future; in this case, the patch of surviving space in a phoenix universe would be infinite all along and would increase in volume by an exponential factor each cycle. An alternative possibility is that the universe had a definite beginning: for example if it was born in an initial quantum creation event, such as in the Hartle-Hawking no boundary proposal [18], or, in the colliding branes picture, through the sudden quantum creation from nothing of a positive- and negative-tension orbifold plane pair with random, but smooth, initial conditions. All that is required is that there be a finite probability for a patch to have the right values of $s$ to grow from cycle to cycle. Even if the branes and/or patch begin small, one ends up with an arbitrarily large, smooth, flat, habitable universe full of matter and radiation as cycles proceed.

The global structure of the phoenix universe is quite different from that of eternal inflation. First of all, there is no amplification of rare quantum fluctuations, as there is for inflation. The phoenix universe, like general cyclic models, has the property that the smoothing phase (dark energy followed by ekpyrotic contraction) has a smaller expansion rate, i.e. a smaller Hubble parameter $H$, than the matter and radiation dominated phases. Hence, if rare quantum fluctuations keep some region in the smoothing phase while typical regions proceed to the matter and radiation dominated phases, the rare regions lose in volume to the typical ones. The situation is reversed with inflation: rare regions kept behind in the smoothing (de Sitter) phase are exponentially enhanced and soon dominate the volume. This principle underlies eternal inflation. The result is that most of the volume in an inflationary universe is empty, uninhabitable and exponentially expanding, punctuated by rare bubbles where inflation has ended, some (but not all) of which are habitable. By contrast, most of the volume in a cyclic universe is habitable.

The phoenix universe adds a new wrinkle because of the instability associated with the entropic mechanism. Namely, only a tiny fraction of the habitable volume in any given cycle survives to the next cycle. Most of the volume fails to make it through the ekpyrotic smoothing phase and collapses through a mixmaster crunch [7] and/or black hole formation (see also [19]). Nevertheless, because the small surviving fraction grows in volume by an exponential factor after the bounce (and the failed regions collapse), most of space during the cycle remains habitable. In this sense, habitability is an attractor in the phoenix universe. Of course, to some extent the present discussion pertains more to metaphysics than to physics - the important question is whether the model discussed here leads to any distinct observational consequences.

One observational signature of great promise has been discussed recently: due to the very steep ekpyrotic potential, ekpyrotic and cyclic models distinguish themselves by a substantial and soon measurable amount of nonGaussianity [20, 21, 22]. A second potentially observable effect is suggested by the above analysis in the case that the cyclic universe has a definite beginning: since the dark energy phase has only just begun, there should still be a significant spread of values of $s$ across the currently observable universe. Hence one would expect the dark energy not to be equal everywhere in space, but to vary by a fractional amount of

$$
\frac{\Delta V}{V} \approx \frac{V_{, s s}(\Delta s)^{2}}{V} \approx \frac{(\Delta s)^{2}}{p} .
$$

Since the spread in values of $s$ satisfies $\Delta s \lesssim p$ and since $10^{-4} \lesssim p \lesssim 10^{-2}$ for models that give observationally acceptable levels of spectral tilt and non-Gaussianity, we would expect a spatial variation in dark energy of

$$
\frac{\Delta V}{V} \lesssim 10^{-4} \text {. }
$$

One would expect to be closer to the upper bound if we are still in one of the early cycles, and less and less variation as we get to older cycles. The largest variation 
occurs for a flat region that was smaller than the horizon size in previous cycles and which exceeds the present horizon size for the first time during the current cycle. However, there is no particular reason to believe that we must be in one of the very early cycles. Nevertheless, conceptually the spatial variation of dark energy can be regarded as a cosmic clock in scenarios where the cyclic universe has a beginning.

Finally, we wish to emphasize the importance of dark energy to the phoenix universe. In previous studies of the cyclic universe based on a single field where there was no instability during the ekpyrotic phase, the role of dark energy was confined to ensuring that the cyclic solution was a stable attractor solution to the equations of motion in the case that the radion overshoots after a bounce (or the branes separate too much after a collision). Dark energy is not needed to smooth or flatten the universe; this is easily accomplished during the ekpyrotic contraction phase alone. To meet the attractor requirement, only two or three e-folds of dark energy domination are needed. For the phoenix universe, though, dark en- ergy plays a new role: it expands the region of space that meets the conditions on $s$ required to complete the unstable ekpyrotic trajectory and rebound as a smooth, flat patch with nearly scale-invariant fluctuations in accord with what is observed. With too few e-folds of dark energy, this patch would shrink from one cycle to the next and would not survive. With at least 60 e-folds of accelerated expansion at the current rate (or 600 billion years of dark energy phase), the patch grows from cycle to cycle, and the phoenix universe is forever reborn. Dark energy is, thereby, given a new role and must satisfy a new constraint. The new constraint is rather mild quantitatively, but, qualitatively, by controlling the survival or termination of the phoenix universe, it may act as a selection criterion that may help to explain why the dark energy density is so small and yet non-zero today [23].

We would like to thank Jim Peebles and Neil Turok for stimulating discussions and comments on the manuscript. This work was supported in part by US Department of Energy grant DE-FG02-91ER40671.
[1] G. Lemaitre, Annales Soc. Sci. Brux. Ser. I A 53, 51 (1933).

[2] E. Komatsu et al. [WMAP Collaboration], arXiv:0803.0547 [astro-ph].

[3] P. J. Steinhardt and N. Turok, Science 296, 1436 (2002).

[4] P. J. Steinhardt and N. Turok, Phys. Rev. D 65, 126003 (2002) arXiv:hep-th/0111098.

[5] J. L. Lehners, Phys. Rept. 465, 223 (2008) arXiv:0806.1245 [astro-ph]].

[6] J. K. Erickson, D. H. Wesley, P. J. Steinhardt and N. Turok, Phys. Rev. D 69, 063514 (2004) arXiv:hep-th/0312009.

[7] D. Garfinkle, W. C. Lim, F. Pretorius and P. J. Steinhardt, arXiv:0808.0542 [hep-th].

[8] P. Horava and E. Witten, Nucl. Phys. B 460, 506 (1996) arXiv:hep-th/9510209.

[9] J. L. Lehners, P. McFadden, N. Turok and P. J. Steinhardt, Phys. Rev. D 76, 103501 (2007) arXiv:hep-th/0702153.

[10] P. Creminelli, A. Nicolis and M. Zaldarriaga, Phys. Rev. D 71, 063505 (2005) arXiv:hep-th/0411270.

[11] R. Durrer, hep-th/0112026 $\quad$ R. Durrer and F. Vernizzi, Phys. Rev. D 66, 083503 (2002); C. Cartier, R. Durrer and E.J. Copeland, hep-th/0301198 R. Brandenberger and F. Finelli, JHEP 0111, 056 (2001); F. Finelli and R. Brandenberger, Phys. Rev. D 65, 103522 (2002); D. Lyth, Phys. Lett. B 524, 1 (2002); Phys. Lett. B 526, 17 (2002); J.C. Hwang, Phys. Rev. D 65, 063514 (2002); P. Peter and N. Pinto-Neto, Phys. Rev. D 66, 063509
(2002); A. J. Tolley, N. Turok and P. J. Steinhardt, Phys. Rev. D 69, 106005 (2004); P. L. McFadden, N. Turok and P. J. Steinhardt, Phys. Rev. D 76, 104038 (2007).

[12] A. J. Tolley and D. H. Wesley, JCAP 0705, 006 (2007) arXiv:hep-th/0703101.

[13] E. I. Buchbinder, J. Khoury and B. A. Ovrut, JHEP 0711, 076 (2007) arXiv:0706.3903 [hep-th]].

[14] J. L. Lehners, P. McFadden and N. Turok, Phys. Rev. D 75, 103510 (2007) arXiv:hep-th/0611259; Phys. Rev. D 76, 023501 (2007) arXiv:hep-th/0612026.

[15] J. L. Lehners and N. Turok, Phys. Rev. D 77, 023516 (2008) arXiv:0708.0743 [hep-th]].

[16] K. Koyama and D. Wands, JCAP 0704, 008 (2007) arXiv:hep-th/0703040.

[17] J. Khoury, P. J. Steinhardt and N. Turok, Phys. Rev. Lett. 92, 031302 (2004) arXiv:hep-th/0307132.

[18] J. B. Hartle and S. W. Hawking, Phys. Rev. D 28, 2960 (1983).

[19] J. K. Erickson, S. Gratton, P. J. Steinhardt and N. Turok, Phys. Rev. D 75, 123507 (2007) arXiv:hep-th/0607164.

[20] K. Koyama, S. Mizuno, F. Vernizzi and D. Wands, JCAP 0711, 024 (2007) arXiv:0708.4321 [hep-th]].

[21] E. I. Buchbinder, J. Khoury and B. A. Ovrut, Phys. Rev. Lett. 100, 171302 (2008) arXiv:0710.5172 [hep-th]].

[22] J. L. Lehners and P. J. Steinhardt, Phys. Rev. D 77, 063533 (2008) arXiv:0712.3779 [hep-th]]; Phys. Rev. D 78, 023506 (2008) arXiv:0804.1293 [hep-th]].

[23] J.-L. Lehners, P.J. Steinhardt and N. Turok, in preparation. 